\title{
Decomposition Removal Technology and General Program Research of Scrap Ammunition
}

\author{
Junsheng Wang ${ }^{1, a}$, Hongjuan Zhu ${ }^{2, b}$, Junwei Lei ${ }^{3, c}$ \\ ${ }^{1}$ the $91872^{\text {th }}$ unit of PLA, Beijing, China \\ 2 Department of naval equipment, Beijing, China \\ ${ }^{3}$ Department of control engineering, Naval aeronautical and astronautical University \\ Yanti, 264001 \\ awangjunshen1024@126.com, 'bhuhongjuan1024@126.com, ${ }^{\mathrm{C}}$ leijunwei@126.com
}

\begin{abstract}
Keywords: ammunition destruction; decomposition; inflammable and explosive; deep ocean dumping; recycling;
\end{abstract}

\begin{abstract}
Scrap ammunition processing activities have a lot of security risks. It is a strong technical and high security requirements professional activities. The decomposition of scrap ammunition is the technical operation process that uses certain technical method to remove the original structure and function of ammunition. The development situation of scrap ammunition, ammunition decomposition programs and decomposition removing requirements are introduced in detail in this paper. The paper has far-reaching significance for the decomposition and removing of scrap ammunition.
\end{abstract}

\section{Introduction}

The decomposition and removal of scrap ammunition is the technical operation process that uses certain technical method to remove the original structure and function of ammunition. The general way of organization is to break down the big first, then decompose the element. The ultimate aim of decomposition is to separate explosive materials or ammunition components containing explosive materials with inert materials or components[1-6]. Technical preparation is done for further processing. Decomposition removal techniques can be used independently, but more are regarded as the basis for subsequent processing. The most prominent advantage of this technique can get more recycled products, even can be recycled or used all components of ammunition. Decomposition removal is the first step of the ammunition destruction, whose components or materials need to deal with. What components can be directly recycled must be determined in the dismantling decomposition stage. Scrap ammunition decomposition removal is mainly restricted by processing sites, facilities, machinery equipment, tools and other material conditions and the technology level of practitioners, as well as the environmental protection laws and explosion safety protection ability. More strict management and organizational procedures are required for scrap ammunition decomposition removal. The processing of scrap ammunition need help of the necessary facilities, through a series of technical methods are used for military purposes of ammunition, after processing, eliminate the danger, and the process of recovery valuable materials. Inflammable and explosive characteristics of ammunition makes the scrap ammunition processing activities have great security risk. It is the professional activities of strong technical and high security requirements[7-11].

Whether a kind of ammunition should be destroyed or not, its life should be analyzed first, so a kind of life distribution concept and statistical analysis method on degradation data is introduced first as follows.

\section{Statistical Analysis of Degradation Data Based on the Pseudo-life Distribution}

Supposing under a stress level, the sample's degradation path obeyed the same life distribution could be described as the curve equation of the same form. Because of the randomness in the samples, the degradation curve equation of the different product samples having different equation 
coefficients[12-13]. Therefore, such random fluctuations make the performance degradation of product reaching a pre-set failure threshold, which the time needed (pseudo failure life) also has a certain degree of randomness. So a kind of distribution could be adopted to describe this randomness of the pseudo failure life[14-17]. And further more the quantitative assessment on the reliability level of product can be done as figure 1.

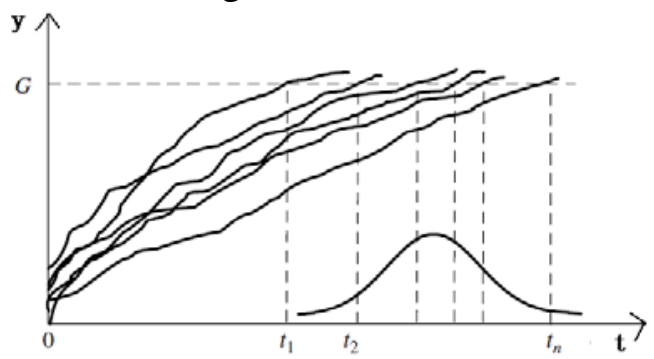

Fig. 1 The relations among degradation paths, the pseudo life and the life distribution

The steps of statistical analysis of degradation data based on pseudo-life Distribution are as follows: (1) Select the model of degradation path; (2) extrapolate the pseudo-life data; (3) execute statistical inference according to the pseudo-life data. This method is the way of approximate degradation analysis. The basic steps are as follows:

(1) Set $n$ samples to be tested, for the sample $i$, with its degradation data $\left(t_{i 1}, y_{i 1}\right), \cdots,\left(t_{i m_{i}}, y_{i m_{i}}\right)$ and degradation failure model $y_{i j}=g_{i j}+\varepsilon_{i j}$, the value $\beta_{i}=\left(\beta_{1 i}, \cdots, \beta_{k i}\right)$ of maximum likelihood estimation can be obtained, denoted by $\hat{\beta}_{i}$.

(2) Solve $t$ using the life equation $g\left(t, \hat{\boldsymbol{\beta}}_{i}\right)=y_{A C}$, denoted as $\hat{t}_{i}$; repeat (1) and (2), then the forecast estimates $\hat{t}_{1}, \hat{t}_{2}, \cdots, \hat{t}_{n}$ of failure data of all the samples can be obtained.

(3) Obtain the failure distribution function $F(t)$ from $\hat{t}_{1}, \hat{t}_{2}, \cdots, \hat{t}_{n}$.

(4) Infer the reliability of product by the function $F(t)$.

Thus, this method increases the pseudo-failure data before the analysis of traditional failure data. Specifically, suppose a degradation test have $n$ samples. During the test, each sample is checked regularly to measure key performance characteristic $Y . y_{i j}$ indicates the measurement values of sample $i$ at $t_{i j}$, here $i=1,2, \cdots, n, j=1,2 \cdots, m_{i}$, and $m_{i}$ is the number of measurements of sample $i$. Degradation trajectory can be expressed as

$$
y_{i j}=g\left(t_{i j} ; \beta_{1 i}, \beta_{2 i}, \cdots \beta_{p i}\right)+\varepsilon_{i j} \square
$$

Here $g\left(t_{i j} ; \beta_{1 i}, \beta_{2 i}, \cdots \beta_{p i}\right)$ is the degradation true value of sample $i$ at $t_{i j}, \quad \varepsilon_{i j}$ is the error term, usually it dependent on $i, j$, and satisfy the normal distribution whose mean is zero and standard deviation is $\sigma_{e}$, and here $\sigma_{e}$ is a constant.

\section{Development Situation of Scrap Ammunition Destruction}

Scrap ammunition disposal is an important research topic around the world. Currently there are many kinds of dealing with waste ammunition. Buried deep, blow up, decomposition method, burning method are more traditional destroy method. With the constant improvement of the requirements for scrap ammunition processing, modern ammunition destruction technology also has made great progress[17-20]. Fusion method, shaped cutting, water cutting, high pressure liquid nitrogen cryogenic cutting method and laser destroy method constantly emerges. The ammunition destruction environment are more security and stability. The environmental pollution is reduced greatly. Until the 1960s, there are two main types of scrap ammunition processing methods: one is the deep sea dumping. The waste ammunition pours into the sea directly. The advantages of this method are simple. The disadvantage is that the ammunition poured into the sea may polluted the 
environment of the sea, affecting fish survival, threat from vessels, but also waste. The other method is open fires or blow up. This method is also simple. It is particularly suited to some that cannot split recycling and unable to process waste ammunition[20-22]. But scrap ammunition are often very batch. Destruction may produce a wide range of explosion, burning. The bigger shock wave may appear. The special field is needed. The pollution of environmental is especially serious. After the '60s, in the case of safe to remove the ammunition, the way of burn or blow up is done for unfavorable to remove the parts and components. The scrap ammunition that technical status is clear is usually put them into small parts by the production process reasoning. The steel, copper, fire explosives are recycled. The fuse and primer are removed to blow up. Small munitions such as bullets, flares or components that technical status is not clear are blown up directly. After the 80s, the processing of scrap ammunition enters the stage of modernization. Its characteristic is that the industrial robot and microcomputer control are used in the destroy of ammunition. In the robot processing line, ammunition is opened and check, sent into decomposition machine by the conveyor, and then received and processed by robots[23].

\section{Remove Programs of Ammunition Decomposition}

Ammunition refers to the device carrying a certain filler such as explosive fire, fire explosive device, pyrotechnic compound, pyrotechnic devices achieving the target killing, demolition, arson and lighting. It is the floorboard of the parts that major in mass ejection way necessary to complete a shooting. Scrap ammunition decomposition removal should strictly in accordance with the relevant technical procedures and general requirements are safe, simple, are advantageous to recycle material. The sensitive and high risk element is removed first, The no-sensitive element is removed second. Ammunition is decomposed into a small element of ammunition, power down, is advantageous to process the dangerous elements further. Containing more ammunition cartridge and projectile is the key element of decomposition removal. The artillery ammunition is very easy to empty. The ammunition is pour out by adopting the manual or automatic way generally. A variety of techniques can be used for decomposition disassemble, such as spiral discharge, cutting, extrusion, heating, air pressure, stamping and so on.

\section{Requirements of Decomposition and Removal}

Scrap ammunition destruction shall comply with the requirements of environmental protection. Avoiding burning waste gas, waste residue of ammunition material impact on the environment as far as possible, eliminating harm to surrounding environment from noise, vibration, shock waves, broken pieces in the destruction process as far as possible. Therefore, the way of direct burning and blow up is not adopted for the batch scrap ammunition generally, but adopting decomposition removal method. The main ingredients of scrap ammunition are steel, aluminum and other metal materials etc. Decomposition removal technology can separate the metal materials with nonmetal materials. The recycling of scrap resources is realized.

The useful resources are recycled maximize. The recycling metal materials can be converted into a variety of civilian goods. The Recycling explosive can be applied to various blasting construction, also can be applied to reload again after refining. Decomposition removal technology makes scrap ammunition become useful resource. Decomposition removal technology is suitable for the batch scrap ammunition destruction.

Decomposition removal technology can reduce the accident risk of scrap ammunition. Scrap ammunition can not meet the needs of combat training, but its inherent combustion and explosion property does not change. The sensitive element has the property of inflammable and explosive such as fuse, electric ignition, primer, the burning and explosion accident can still be caused by the stimulation of external energy. Decomposition removal technology can remove sensitive element such as ammunition fuze, primer. The sensitivity of the ammunition is reduced. The safety of transport and destruction scrap ammunition is improved. Decomposition removal technology can separate launch canister from the projectile. Ammunition independent components are good for 
safety management and further processing. The removable sex of ammunition is the comprehensive evaluation of remove the ease ammunition. Removable of ammunition is researched systematically.

The optimal way and sequence of disassemble is found out by the basic information of the ammunition and its components, security, technical feasibility, environmental hazards etc. Ammunition decomposition disassemble is usually carried out in accordance with inverse order of the ammunition production. Ammunition disassemble is destructive irreversible process. The energy consumption, the cost of remove and impact on the environment need to be considered for the removal of ammunition. The removal can be done by professional decomposition equipment. Disassemble of scrap ammunition is a highly dangerous work. It is implemented in strict accordance with the procedure by using specialized equipment and tools. Scrap ammunition disassemble process planning is implemented after the supervisor for approval by experts familiar with structure and processing technology of ammunition. The decomposition process of scrap ammunition should be strictly through the following procedures. Collecting scrap ammunition information, analysis of ammunition, determining the decomposition removal depth, drawing the disassemble parts diagram, simulating decomposition disassemble etc. Ammunition decomposition process is a legal technical files, the operator must comply with them. The operator should study ammunition disassemble process in detail, understand ammunition structure and material properties, particularly be familiar with toxicity and pollution characteristics of explosive materials, do a good job of safety according to safety, environmental protection, recycling before decomposition ammunition work.

\section{Conclusion}

The decomposition and removal of scrap ammunition is the technical operation process that uses certain technical method to remove the original structure and function of ammunition. Compared with scrap ammunition processing development present situation of some developed countries, we are still a gap in technical level, systematic and series. The development situation of scrap ammunition, ammunition decomposition programs and decomposition removing requirements are introduced in detail in this paper.

The paper has very important significance for the decomposition and removal of scrap ammunition.

\section{References:}

[1] Zhu Shao-wu, Xie Xian-qi, Luo Qi-jun. The design and practice of shells and expired blasting equipment destruction[J]. Blasting,2001,6(2):87-89.

[2] Wen Jian-ping, Hu Xiao-long, Wei Xiao-lin. The design and implementation of explosion method to destroy waste shells in cave[J]. Blasting,2007,24(3):84-87.

[3] Zhang Ke-ling, Xu Chun. Identifying and destruction equivalent estimate of the shells[J]. Blasting,2003, 6(2):17-21.

[4] Wang Dong-sheng, Wang Fang-xiao. Related experience of explosion operations from outdoor to indoor[J].Journal of safety and environment,2004,4:122-123.

[5] Fu Wu,Wang Jian,Fu Bo. Shaft kiln and kiln body building directional blasting demolition at the same time under complicated environment[J]. Egineering blasting,2010,16(2):53-56.

[6] Zhou Jia-han. The discussion of blasting demolition vibration velocity calculation formula[J]. Engineering blasting, 2009,26(1):37-41.

[7] Zhu Chao-xiang,Cui Yun-wu,Gao Xing. Blasting demolition of two $120 \mathrm{~m}$ high reinforced concrete chimneys[J]. Engineering blasting[J].2008,14(2):61-63.

[8] Cui Xiao-rong,Wang Dian-guo,Lu Hua. Directional blasting demolition of hundreds meters 
reinforced concrete chimney[J]. Blasting,2008,25(4):56-58.

[9] Guo Tao, Qi Shi-fu, Wang Shu-min,Ding Wen. The application of large quantities of waste ammunition blasting destruction technology[J]. Engineering blasting,2011,17(2):89-91.

[10] Zhang Ke-ling,Xu Chun. The safety distance calculation of aerial bomb destruction[J]. Blasting,2003(4): 24-26.

[11] Li Shi-chong. The destruction of war times legacy waste ammunition[J]. Coastal enterprises and science technology,2007,7:183-186.

[12] Zhou Ming-an, Xia Jun, Xiao Zhi-wu. Inspection and destruction discussion of war explosive remnants[J].Blasting, 2007, 24( 2) : 82-86.

[13] Zhai Xin-fu, Ying An-ming, Nie Cheng-yue. The safety issues and countermeasures in the process of waste ammunition destruction[J]. Journal of sichuan armaments factories, 2002, 23(4):36-37.

[14] Li Jin-ming, Gao Xin-bao,Ding Yu-kui. Accident prevention measures in the process of scrap ammunition blasting destruction[J].Blasting, ,2011,28(3):116-118.

[15] Yi Jian-kun, He Wu-yi,Wu Teng-fang. High hot agent application in the field of ammunition destruction[J]. Engineering blasting,2004,10(4):21-25.

[16] Zhang Ke-ling, Xu Chun. Identifying and destruction equivalent estimate of the waste shells[J]. Blasting, 2003,20(2): 80-82.

[17] Ding Yu-kui, Li Jin-ming. The research of conventional ammunition destruction method[A]. The 9th national academic conference proceedings on explosion and safety technology[C],Shen Yang: china ordnance society,2006 :83 - 86.

[18] Wang Dao-quan, Lv Guo-bin, Hao Bin. The method of using anti-explosion room destruction detonator[J]. Engineering blasting,2002,8(3):74-76.

[19] Ge Yong, Fu Tian-guang,Yang Zhi-guang. Energy-gathered cut destruction waste shells[J]. Engineering blasting, 2006,12(4):74-76.

[20] Jiang Yue-fei, Zhang Zheng-zhong, Yang Yan, Wang Zhong-guo. The design and construction of scrap ammunition destruction[J]. Engineering blasting,2019,15(2):87-90.

[21] Luo Zhi-li. Several realizations of disposal war legacy shells[J]. The guangdong public security science and technology, 2004,(4):64-66.

[22] Lou Jian-wu, Long Yuan, Xie Xing-bo. Disposal and destruction technology of abandoned explosives and conventional ammunition[M]. Beijing: National Defence Industry Press, 2007:155-228.

[23] Hu Zhen,Liu Chu-ping, Shu Fu-gui. The research of thermal plasma technology destruction Japanese abandoned chemical weapons filler[J]. Journal of safety and the environment, 2006, 6(6):84-87. 